\title{
Control basado en pasividad con estrategia adaptativa de un sistema de almacenamiento energético para una nano-red $D C^{*}$
}

\author{
Sebastián Sánchez-Choachi"* \\ Recibido: 26/09/2017 • Aceptado: 11/02/2019 \\ https://doi.org/10.22395/rium.v18n35a11
}

\begin{abstract}
Resumen
El creciente interés en el desarrollo de las micro-redes ha impulsado un gran número de investigaciones que pretenden mejorar su desempeño para una posterior industrialización. Por este motivo, este artículo presenta el modelamiento, diseño de control y resultados de simulación de un sistema de almacenamiento energético para una nano-red DC, el cual permite almacenar energía en las baterías o extraerla de ellas, según sea el estado de las cargas y las fuentes de la nano-red. Este dispositivo se construye a partir de un convertidor bidireccional DC-DC no aislado, para el que se desarrolla su modelo y posterior estrategia de control basada en pasividad, acompañada de un mecanismo adaptativo que le permite estimar algunos de sus parámetros y cambios. Se presentan los resultados de simulación entre los cuales destaca la rápida respuesta del sistema, su robustez ante perturbaciones y la contribución a la regulación de la tensión del bus DC de la nano-red.
\end{abstract}

Palabras clave: cargador de baterías; convertidores de potencia DC-DC; estimación de parámetros; fuentes de alimentación de emergencia; nano-red DC; sistema adaptativo.

\footnotetext{
Este artículo es el resultado de la investigación en curso del proyecto Cargador de baterías de mediana y baja capacidad con baja distorsión armónica en corriente, elevado factor de potencia y alta eficiencia para vehículos eléctricos (113071551746), financiado por Colciencias durante el tiempo comprendido entre el 8 de abril del 2016 y el 8 de abril del 2018.

** Ingeniero electrónico y físico, magíster en Ingeniería Electrónica. Investigador activo del grupo LIfae, Universidad Distrital Francisco José de Caldas, Bogotá, Colombia. Correo electrónico: jossanchezc@correo. udistrital.edu.co. Orcid: https://orcid.org/0000-0002-1945-5975
} 


\title{
Passivity-Based Control with an Adaptive Strategy of an Energetic Storage System for a DC Nano Grid
}

\begin{abstract}
The growing interest in the development of micro-grids has led to a large number of research projects, in order to improve their performance for further industrialization. For this reason, this paper presents the modeling, control design and simulation results of an energy storage system for a DC nano-grid. This device allows storing energy in the batteries or extract it from them, depending on the load and state of the nano-grid sources. This equipment is built from a nonisolated DC-DC bidirectional converter, for which its model and control strategy based on passivity, accompanied by an adaptive mechanism that estimates some parameters of the system are developed. The simulation results are presented, highlighting the fast response of the system, its robustness facing disturbances and its contribution to the regulation of the DC bus voltage of the nano-grid.
\end{abstract}

Keywords: Adaptive system; battery chargers; DC-DC power converters; DC nano-grid; emergency power supplies; parameter estimation.

\section{Controle baseado em passividade com estratégia adaptativa de um sistema de armazenamento energético para uma nanorrede $D C$}

\begin{abstract}
Resumo
$\mathrm{O}$ crescente interesse no desenvolvimento das microrredes impulsionou muitas pesquisas que pretendem melhorar seu desempenho para uma posterior industrialização. Por esse motivo, este artigo apresenta a modelagem, desenho de controle e resultados de simulação de um sistema de armazenamento energético para uma nanorrede DC, que permite armazenar energia nas baterias ou extraí-la das baterias, de acordo com o estado das cargas e das fontes da nanorrede. Esse dispositivo é construído a partir de um conversor bidirecional DC-DC não isolado, para o qual se desenvolve o modelo e posterior estratégia de controle baseada em passividade, acompanhada de um mecanismo adaptativo que o permite estimar alguns de seus parâmetros e alterações. Apresentam-se os resultados de simulação entre os quais se destaca a rápida resposta do sistema, sua robustez ante perturbações e a contribuição para a regulação da tensão do ônibus DC da nanorrede.
\end{abstract}

Palavras-chave: carregador de baterias; conversores de potência DC-DC; estimativa de parâmetros; fontes de alimentação de emergência; nanorrede DC; sistema adaptativo. 


\section{INTRODUCCIÓN}

En la actualidad se desarrollan múltiples investigaciones en torno a las micro-redes como consecuencia de ventajas tales como la reducción de pérdidas de transmisión y distribución, junto con el beneficio ecológico que tiene este tipo de sistemas al incluir dentro de su estructura fuentes alternativas como la eólica y fotovoltaica. Esto permite además, diversificar las fuentes de generación eléctrica, que en países como Colombia son mayormente hídricas [1], lo cual hace difícil y costoso la expansión de su capacidad, y causa una alta dependencia de fenómenos naturales, lo que a su vez genera una relación directa entre el precio de la energía y el caudal de los ríos [2] y pone en riesgo el sector eléctrico y todos los demás que directa e indirectamente dependen de este. Adicionalmente, la implementación de este tipo de sistemas también ofrece acceso al fluido eléctrico en las zonas que no se encuentran conectadas a la red eléctrica, lo que supone un gran número de beneficios económicos y sociales para cada una de esas zonas.

Las micro-redes que desarrollan capacidades inferiores a $5 \mathrm{~kW}$ se denominan normalmente nano-redes, las cuales tienen aplicaciones en sistemas de comunicaciones y de oficina [3], o domésticas y de entretenimiento [4]. A su vez, las nano-redes se pueden clasificar en $\mathrm{AC}$ o DC, siendo estas últimas sobre las cuales se centra el presente trabajo, esto debido a sus ventajas frente a las primeras, tales como simplicidad en diseño y control, mayor eficiencia al no poseer convertidores AC-DC y DC-AC para conectar las fuentes de generación renovable, bajas pérdidas de transmisión debido a la cercanía con las fuentes alternativas de generación y la ausencia de problemas de sincronismos o distorsión armónica [5], con lo cual se evita el uso de complejos correctores de factor de potencia como el desarrollado en [6], en el que se evidencia una notable complejidad, problemas de funcionamiento e incremento del costo de implementación de la nano-red.

A pesar de las ventajas ya mencionadas, las nano-redes en modo isla son vulnerables a cambios climáticos debido al uso de fuentes eólicas y fotovoltaicas, lo que les impide ofrecer un suministro eléctrico ininterrumpido por sí solas. Por tal motivo, se incluyen los sistemas de almacenamiento energético (SAE), que responden al estado conjunto de las fuentes y las cargas almacenando energía cuando las fuentes generan más de la que consumen las cargas y entregándola en caso de que las condiciones climáticas no permitan suplir la potencia demandada por el bus DC. Adicionalmente, los SAE contribuyen a la regulación de los niveles de tensión y mejoran la estabilidad del sistema [7]. Por estos motivos, resulta evidente la importancia de los SAE dentro del funcionamiento de la nano-red, lo que sugiere dedicar especial atención al mejoramiento de los sistemas de almacenamiento energético como estrategia de robustecimiento de las nano-redes. 
Estos SAE están compuestos por un conjunto de baterías que se conectan al bus DC mediante un convertidor bidireccional que ajusta los niveles de tensión. Dicho convertidor debe ser eficiente y operar de la manera en la que se ilustra en la figura 1, en la que la tensión de referencia $\left(v_{\text {cref }}\right)$ divide la región de operación en dos. En la región superior, el SAE almacena energía y en la inferior, la extrae de las baterías y la inyecta al bus DC de acuerdo con las necesidades de las cargas.

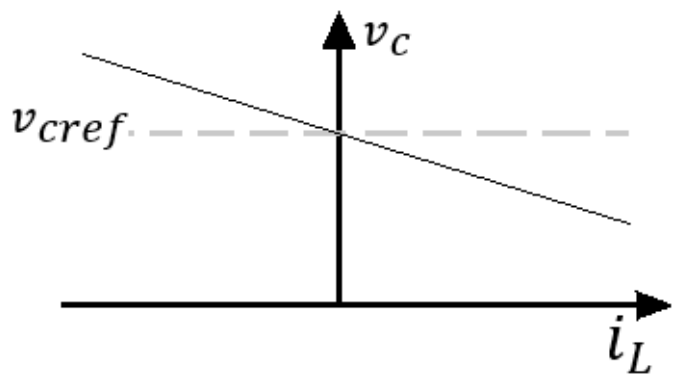

Figura 1. Corriente del SAE de acuerdo con la tensión del bus DC

Fuente: elaboración propia

Se pueden emplear diferentes topologías de conversión junto con distintas estrategias de control para el desarrollo de sistemas de este tipo. Dentro de las estrategias de control es posible encontrar controladores lineales como los tradicionales PID y controladores no lineales como modos deslizantes [8], controladores difusos [9], TCM [10] y finalmente, los basados en pasividad como el mostrado en [11]. Todos estos controladores presentan problemas al incorporar incertidumbre en algunos de sus parámetros, sin embargo, el controlador basado en pasividad es especialmente sensible a estos cambios, como lo ratifica [11] al establecer como una de las condiciones de funcionamiento del sistema que "todos los parámetros son totalmente definidos y bien conocidos", lo cual en la práctica es difícil de lograr, dada la imposibilidad de tener total certeza de estos parámetros o por la variación de estos frente a diferentes condiciones del sistema.

No obstante, en trabajos como el presentado en [12], se incorporan técnicas de adaptabilidad mediante la identificación algebraica de parámetros, en la que se usan ventanas de tiempo relativamente grandes para la determinación de dichos parámetros y en los cuales se asume que dicha variable permanece constante, lo que lo convierte en un método de largos tiempos de respuesta. Por lo anterior, este articulo presenta el modelamiento y diseño de control de un SAE para una nano-red DC, que a diferencia de los muchos desarrollos al respecto, hace uso de una topología de conversión no aislada de alta eficiencia, cuyo control se basa en pasividad, lo cual asegura una estabilidad ininterrumpida del sistema, pero que presenta problemas de robustez al poseer un alto 
grado de dependencia de los parámetros del sistema. Para solucionar este problema, se incorpora un mecanismo de adaptabilidad basado en inmersión e invariancia que permite estimar estos parámetros mediante la lectura de corriente y tensión, además del conocimiento de la dinámica del sistema.

\section{MODELAMIENTO}

Por razones de rendimiento, simplicidad y eficiencia, se elige al convertidor reductorelevador mostrado en la figura 2, como el encargado de permitir el intercambio bidireccional de energía entre la batería y el bus DC, cuyo modelo también es presentado en esa misma figura. Este convertidor opera como un elevador del puerto 1 al 2 si se conmuta S1, mientras S2 está abierto, con lo que solo opera su diodo de cuerpo. Para el caso contrario en el que S2 conmuta y $\mathrm{S} 1$ está abierto, el convertidor operará como un reductor del puerto 2 al 1 . En consecuencia, la señal de conmutación del interruptor $\mathrm{S} 1$ es la misma que la del interruptor S2, pero negada, lo que permite tener un modelo único del sistema y no uno para cada modo de operación, que funcionará dependiendo del estado del bus DC, que para este caso es representado como el paralelo entre una carga resistiva y una fuente ideal de corriente.

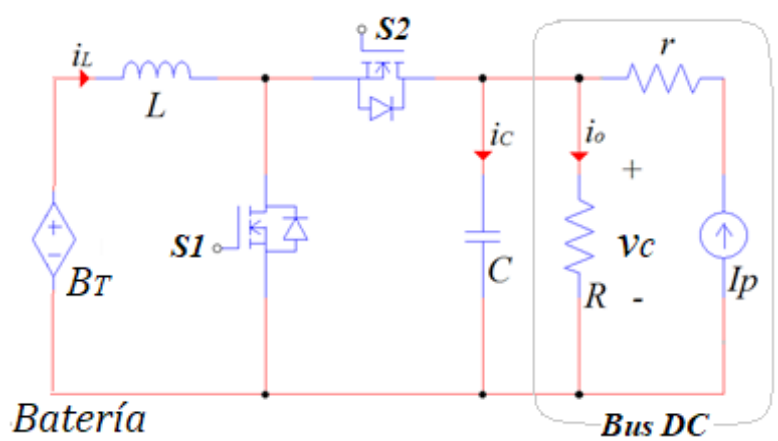

Figura 2. Modelo de circuito del SAE y el bus DC

Fuente: elaboración propia

\subsection{Modelo del SAE}

Para caracterizar el comportamiento del convertidor, se hará uso del formalismo de Euler-Lagrange, en el que el sistema se lleva a la forma indicada por la ecuación 1, siendo $\boldsymbol{X}$ la matriz columna de las variables estado del sistema, que para este caso son la corriente en el inductor $\left(i_{L}\right)$ y la tensión del condensador de salida $\left(v_{c}\right)$ que se encuentra conectado al bus DC, $d$ es el ciclo útil, $\boldsymbol{H}$ es una matriz definida positiva que relaciona la energía almacenada en los elementos, $\boldsymbol{F}$ considera la energía interna del sistema y finalmente $\boldsymbol{K}$ es una matriz definida como positiva que da cuentas de la razón de disipación de energía. 


$$
\boldsymbol{H} \dot{\boldsymbol{X}}=(\boldsymbol{F}(d)+\boldsymbol{K}) \boldsymbol{X}-\boldsymbol{G}\left(B_{T}\right)
$$

Al desarrollar las ecuaciones de malla del convertidor para los dos estados de los interruptores S1 y S2, se obtiene la ecuación 2, que ha sido promediada en una ventana de tiempo de igual duración al tiempo de conmutación, y ajustada para que corresponda con la forma indicada en la ecuación 2. Esta ecuación conserva la misma simbología mostrada en la figura 1. En esta figura se observa que la batería usada es modelada simplemente como una fuente de tensión, sin embargo, se ha de aclarar que esta posee su propia dinámica y puede ser representada mediante modelos más completos en los que predomina su resistencia interna. Esta resistencia es un parámetro dinámico que depende de diferentes factores como el estado de carga de la batería [13] y su corriente instantánea [14], razón por la cual no sería conveniente en la práctica incluirla en el modelo. No obstante, esto no resulta en una falencia del trabajo desarrollado, sino en todo lo contrario, pues con la estrategia de adaptabilidad adoptada, la tensión en los terminales de la batería se estima todo el tiempo, lo que hace que sea posible ignorar la tensión que cae en su resistencia interna sin detrimento del desempeño del convertidor.

$$
\left(\begin{array}{ll}
L & 0 \\
0 & C
\end{array}\right)\left(\begin{array}{l}
\frac{d i_{L}}{d t} \\
\frac{d v_{c}}{d t}
\end{array}\right)=\left(\begin{array}{cc}
0 & -(1-d) \\
1-d & 0
\end{array}\right)\left(\begin{array}{l}
i_{L} \\
v_{c}
\end{array}\right)+\left(\begin{array}{cc}
0 & 0 \\
0 & -1 / R
\end{array}\right)\left(\begin{array}{l}
i_{L} \\
v_{c}
\end{array}\right)+\left(\begin{array}{l}
B_{T} \\
i_{P}
\end{array}\right)
$$

Del mismo modo, se selecciona el modelo del bus DC como la combinación en paralelo de una fuente de corriente junto con una carga, en lugar de su equivalente con fuente de tensión porque de esta manera resulta evidente el flujo de energía entre el SAE y el bus DC.

Se ha de recordar que el objetivo principal es controlar la tensión de salida del SAE, sin embargo, al operar en modo elevador, el convertidor exhibe un comportamiento de fase no mínima, razón por la que no es posible realizar un control directo basado en pasividad de la tensión de salida. Para solventar este problema se elige la corriente en el inductor como la variable de control directa y se relaciona con la tensión de salida a través de sus valores de equilibrio mediante la ecuación 3, la cual se puede obtener a partir de la ecuación 2 al fijar en cero las derivadas de las variables de estado, para luego despejar los valores de equilibrio de las variables del sistema.

$$
i_{\text {Lref }}=\frac{v_{\text {cref }}^{2}}{R B_{T}}-\frac{i_{P} v_{\text {cref }}}{B_{T}}
$$




\subsection{Diseño del controlador}

Antes de iniciar con el diseño del control es necesario establecer si es posible un control basado en pasividad con la corriente en el inductor como variable de control. Para esto se determina el grado relativo de la función de transferencia entre el ciclo útil $(d)$ y la corriente en el inductor, que en este caso es de 1, siendo este valor igual al tamaño del vector de control, lo que posibilita el desarrollo del controlador. Una vez verificada la realizabilidad del control, se procede a su desarrollo, que consiste en separar la dinámica del sistema de la siguiente manera $\boldsymbol{X}=\left(X_{L} X_{C}\right)$, en la que $X_{C}$ hace referencia a las variables a controlar de forma directa, mientras que $X_{L}$ contiene los llamados modos libres, que son las variables de estado libres del sistema. De esta forma, la dinámica de las variables controladas y libres se encuentra regida por las ecuaciones 4 y 5 respectivamente, en las cuales se ha añadido el subíndice " $C$ " para notar todas las variables relacionadas con la dinámica a controlar y el subíndice " $L$ " para los términos relacionados con los modos libres.

$$
\begin{gathered}
G_{C}\left(d, B_{T}\right)-\left[\left(F_{C}(d)+K_{C}\left(u_{c}\right)\right) i_{\text {Lref }}+F_{C L} v_{P}\right]+K_{i C}\left(i_{L}-i_{\text {Lref }}\right)=0 \\
G_{L}\left(d_{c}, B_{T}\right)-\left[H_{L} \frac{d v_{P}}{d t}+\left(F_{L}\left(u_{c}\right)+K_{L}\right) v_{P}+F_{L C} i_{\text {Lref }}\right]+K_{i L}\left(v_{c}-v_{P}\right)=0
\end{gathered}
$$

La identificación de cada uno de los términos de las ecuaciones (4) y (5) se lleva a cabo mediante el uso de las ecuaciones 1 y 2 , de forma tal que el resultado de aplicar las ecuaciones 4 y 5 al convertidor bidireccional, se muestra en la ecuación 6 para la dinámica controlada y en la ecuación 7 para los modos libres, siendo $i_{\text {Lref }}$ el valor de referencia elegido para la corriente a través de la ecuación 3 y el valor fijado para la tensión de salida.

$$
\begin{gathered}
B_{T}-\left[(0+0) \cdot i_{\text {Lref }}+(1-d) v_{P}\right]+K_{\text {iC }}\left(i_{L}-i_{\text {Lref }}\right)=0 \\
i_{P}-\left[C \frac{d v_{P}}{d t}+\left(0+\frac{1}{R}\right) v_{P}+(d-1) i_{\text {Lref }}\right]+K_{i L}\left(v_{c}-v_{P}\right)=0
\end{gathered}
$$

A partir de la ecuación 6 es posible despejar el valor de la señal de control $d$ y ponerla en términos de los parámetros del sistema, la variable libre $v_{P}$ y la ganancia del controlador $K_{i C}$, cuyo valor permite ajustar la respuesta del sistema. La expresión derivada de este procedimiento se presentada en la ecuación 8 . 


$$
d=1-\frac{K_{i C}\left(i_{L}-i_{\text {Lref }}\right)+B_{T}}{v_{P}}
$$

Se ha de aclarar que, debido a su definición, $d$ tiene valores restringidos conceptualmente en el rango de 0 a 1 , por lo que se hace conveniente que a través de $K_{i C}$ la ecuación 8 esté restringida a este rango de operación para así evitar efectos de saturación. Luego de la ecuación 7 se obtiene la dinámica de la variable libre $v_{c}$ a través de la variable $v_{P}$, y se generan la ecuación 9.

$$
C \frac{d v_{P}}{d t}=(1-d) i_{\text {Lref }}-\frac{v_{P}}{R}+K_{i L}\left(v_{c}-v_{P}\right)+i_{P}
$$

El uso de cada una de las ecuaciones anteriormente desarrolladas dentro de la estructura de control se ilustra en la figura 3. En esta se ve cómo la ecuación 3 se utiliza para transformar la referencia de tensión en la referencia de corriente, lo que permite usar esta última como la variable directa de control. Luego, este valor de referencia se emplea en el bloque que desarrolla la dinámica de la variable libre $v_{P}$, que además hace uso de la lectura de la tensión de salida, junto con el valor del ciclo útil $d$, obtenido del bloque de la ecuación 8 que depende del valor de la corriente del inductor, la corriente de referencia y la variable libre.

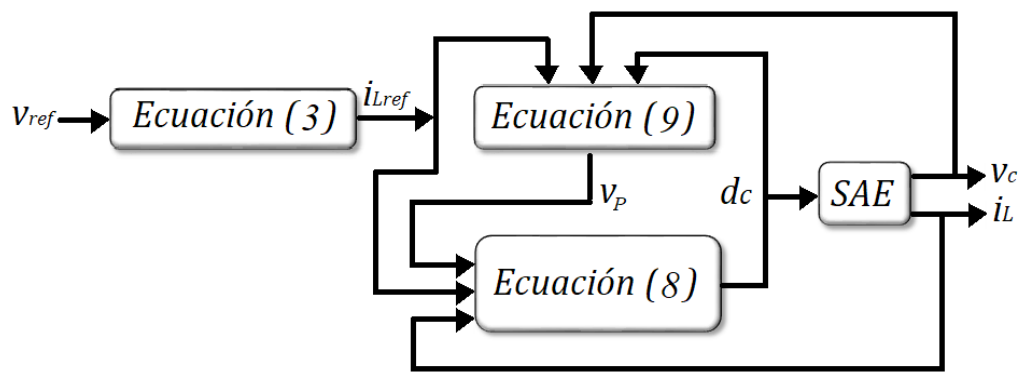

Figura 3. Esquema de control basado en pasividad para el SAE

Fuente: elaboración propia

Una vez desarrollada la estructura de control, se debe sintonizar a través de la elección de las ganancias $K_{i L}$ y $K_{i C}$. Para esto se establecen los límites dentro de los que deberían estar estas ganancias para un correcto funcionamiento, y por ello se establecen los rangos de los tiempos de respuesta de las variables del sistema; iniciando con la corriente del inductor cuya constante de tiempo debe ser superior al tiempo de la ventana de conmutación $\tau_{s}$, tal como lo establece la ecuación 10 .

$$
\tau_{C}>\tau_{s}
$$


Como se evidencia en la figura 3, el bloque que genera la señal de control depende de la dinámica de la variable libre, lo cual obliga a que esta última variable tenga tiempos de respuesta más rápidos que los de la variable a controlar. El criterio usado en este desarrollo será el que muestra la ecuación 11, en la cual se fija la constante de tiempo de la variable libre como por lo menos cinco veces la constante de tiempo $\tau_{C}$.

$$
\tau_{L}>5 \tau_{C}
$$

Para relacionar estas constantes de tiempo con las ganancias $K_{i L}$ y $K_{i C}$, junto con los demás parámetros del sistema se toma la ecuación 8 y se reemplaza en la primera fila de la ecuación matricial 2, para obtener como resultado la ecuación 12.

$$
L \frac{d i_{L}}{d t}=K_{i c}\left(i_{L}-i_{L r e f}\right)
$$

De esta se concluye que la constante de tiempo de esta variable está dada por la ecuación 13.

$$
\tau_{C}=\frac{L}{K_{i C}}
$$

En cuanto a la constante de tiempo de la variable libre, se debe evaluar la ecuación 9 asumiendo que el objetivo del controlador se ha cumplido y las variables libre y de control se encuentran desacopladas, lo que permite tomar $d$ como una constante y establecer el valor de la constante de tiempo para la variable libre $\left(\tau_{L}\right)$ según la ecuación 14.

$$
\tau_{L}=\frac{C}{1 / R+K_{i L}}
$$

Al reemplazar las ecuaciones 13 y 14 en las ecuaciones 10 y 11 respectivamente, se obtienen las expresiones 15 en las que se restringe el valor máximo de las ganancias del controlador, al recordar que estas ganancias deben ser positivas y diferentes a cero.

$$
K_{i c}<\frac{L}{\tau_{s}} \quad K_{i L}<\frac{C K_{i c}}{10 L}-\frac{1}{R}
$$

En este punto, es necesario asegurar que el sistema, ahora compuesto por tres variables, sea estable alrededor del punto de equilibrio fijado a conveniencia, por ello se construye el modelo matemático del sistema aumentado de la figura 3, en la que se incluye la variable $v_{P}$, para obtener así la ecuación de estado (16). 


$$
\left(\begin{array}{c}
\dot{i_{L}} \\
\dot{v_{C}} \\
\dot{v_{P}}
\end{array}\right)=\left(\begin{array}{ccc}
0 & (d-1) / L & 0 \\
(1-d) / C & -1 / R C & 0 \\
0 & K_{i L} / C & -\left(\frac{1}{R}+K_{i L}\right) / C
\end{array}\right)\left(\begin{array}{l}
i_{L} \\
v_{C} \\
v_{P}
\end{array}\right)+\left(\begin{array}{c}
B_{T} / L \\
i_{P} \\
\left((1-d) i_{\text {Lref }}+i_{P}\right) / C
\end{array}\right)
$$

En el modelo de la ecuación 16 el valor del ciclo útil $(d)$ se debe reemplazar con la expresión fijada por la ecuación 8 , cuya estructura depende de otras variables de estado del convertidor y del controlador. Esta sustitución hace que el modelo del sistema deje de ser bilineal, por lo que dicho modelo se debe linealizar, y para ello se desarrolla el Jacobiano de la matriz que multiplica el vector de estados, con la sustitución mencionada, y se evalúa en su punto de equilibrio, que para este caso corresponde a $i_{L}=i_{\text {Lref }}, v_{c}=v_{\text {cref }} \mathrm{y} v_{P}=v_{\text {cref }}$. La ecuación 17 muestra este Jacobiano.

$$
\left(\begin{array}{ccc}
-\frac{K_{i C}}{L} & -\frac{B_{T}}{v_{\text {cref }} L} & \frac{B_{T}}{\left(B_{T}+v_{\text {cref }}\right) L} \\
\frac{B_{T}}{v_{\text {cref }} C}+\frac{K_{i C} v_{\text {cref }}^{2}}{B_{T} R C\left(B_{T}+v_{\text {cref }}\right)} & -\frac{1}{R C} & \frac{-v_{\text {cref }}^{2}}{R C\left(B_{T}+v_{\text {cref }}\right)^{2}} \\
\frac{K_{i C} v_{\text {cref }}^{2}}{B_{T} R C\left(B_{T}+v_{\text {cref }}\right)} & \frac{K_{i L}}{C} & -\frac{1}{C}\left(\frac{1}{R}+K_{i L}+\frac{v_{\text {cref }}^{2}}{R\left(B_{T}+v_{\text {cref }}\right)^{2}}\right)
\end{array}\right)=A_{J}
$$

El valor numérico de cada uno de los parámetros del convertidor en construcción se presenta en la tabla 1 , en la cual destacan los altos valores de $L$ y $C$ que se justifican debido a que se eligió como parámetro de diseño un rizo de corriente de $5 \%$ y tensión del $2 \%$.

Tabla 1. Parámetros eléctricos del sistema de almacenamiento energético

\begin{tabular}{lcccccc}
\hline$L[\mu H]$ & $C[\mu F]$ & & $B_{T}[V]$ & $R[\Omega]$ & $v_{\text {cref }}[V]$ & $P[W]$ \\
\hline 100 & 100 & 12 & 10 & 48 & 230 \\
\hline \multicolumn{6}{c}{ Fuente: elaboración propia }
\end{tabular}

Al reemplazar en la ecuación 17 los valores consignados en la tabla 1 se pueden obtener los valores propios de $A_{J}$, que son a su vez los polos del sistema de la figura 3. Debido a que el valor de estos polos es dependiente de $K_{i C}$ y $K_{i L}$, la parte izquierda de la figura 4 muestra la ubicación de los polos al variar $K_{i C}$, mientras se fija el valor de $K_{i L}$. De igual forma, el resultado de fijar el valor de $K_{i C}$ mientras se varía el valor de $K_{i L}$ se muestra en la parte derecha de esta figura. Todo esto, teniendo en cuenta los límites establecidos por la ecuación 15 . 

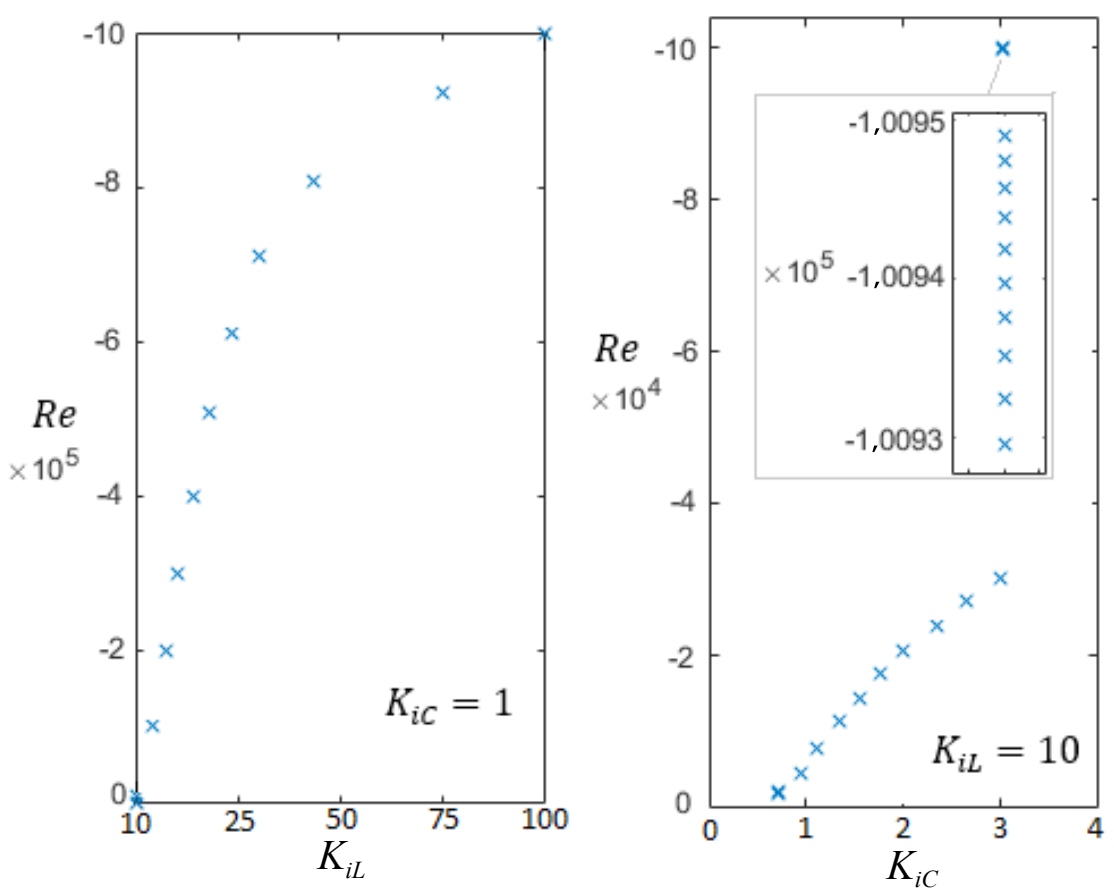

Figura 4. Polos del SAE para diferentes valores de ganancias del controlador

Fuente: elaboración propia

La primera conjetura que se puede realizar de la figura 4 es que, independientemente de la magnitud de las ganancias del controlador, todos los polos del sistema son reales negativos puros, lo cual asegura la estabilidad del SAE y además sugiere transitorios sin sobrepicos, lo cual resulta conveniente para la regulación del bus DC. De igual forma, en esta figura se observa que al aumentar los valores tanto de $K_{i C}$ como de $K_{i L}$, los polos se desplazan cada vez más hacia el semieje negativo, lo que hace que los márgenes de estabilidad del sistema incrementen y los tiempos de respuesta disminuyan, como ya lo mostraban las ecuaciones 13 y 14 .

\subsection{Estrategia de adaptabilidad}

Si bien se completó el diseño del controlador basado en pasividad, como se menciona en la introducción y se evidencia en las ecuaciones 3,8 y 9, este controlador es fuertemente dependiente de los parámetros del convertidor, tales como el voltaje de la batería y el valor de la carga, lo que le da un bajo grado de robustez y alta vulnerabilidad a cambios en el sistema. Por este motivo, al controlador ya diseñado se le añade una estrategia adaptativa que le permite estimar los parámetros necesarios para su funcionamiento 
a partir de las lecturas de tensión de salida y corriente en el inductor. La forma de estimar dichos parámetros se da mediante el uso de inmersión e invariancia, técnica que se explica y aplica al SAE a continuación.

Este procedimiento inicia con la construcción matemática del estimador de la tensión en la batería $\left(B_{T E}\right)$, cuya estructura seleccionada es $B_{T E}=\alpha_{E}+\beta_{E}\left(i_{L}\right)$, en la que se elige que $\beta_{E}$ sea dependiente únicamente de $i_{L}$, pues de acuerdo con la ecuación 2 , la dinámica de esta variable se encuentra relacionada con el valor del voltaje de la batería $B_{T}$. Por consiguiente, el error de estimación $\left(z_{E}\right)$ está dado por la ecuación 18.

$$
z_{E}=\alpha_{E}+\beta_{E}\left(i_{L}\right)-B_{T}
$$

Ahora se considera la dinámica del error de estimación, para ello se toma la derivada temporal de la ecuación 18 para llegar a la ecuación 19.

$$
\frac{d z_{E}}{d t}=\frac{d \alpha_{E}}{d t}+\frac{d \beta_{E}}{d i_{L}} \frac{d i_{L}}{d t}-\frac{d B_{T}}{d t}
$$

Si se considera que los cambios en la tensión de la batería son lentos con respecto a la dinámica del error $\left(z_{E}\right)$, se puede asumir que $d B_{T} / d t=0$. Luego, si se reemplaza el valor de la derivada de $i_{L}$ obtenida de la ecuación 2 , se llega a la ecuación 20 , en la cual además se usó el valor de $B_{T}$ de la ecuación 18 .

$$
\frac{d z_{E}}{d t}=\frac{d \alpha_{E}}{d t}+\frac{d \beta_{E}}{d i_{L}}\left(\frac{d-1}{L} v_{c}+\alpha_{E}+\beta_{E}-z_{E}\right)
$$

Por conveniencia se elige que la derivada temporal de $\alpha_{E}$ anule todos los términos que no acompañen al error $z_{E}$, con lo cual la expresión resultante se muestra en la ecuación 21.

$$
\frac{d z_{E}}{d t}=-z_{E} \frac{d \beta_{E}}{d i_{L}}
$$

Para que el error posea una dinámica estable, se debe asegurar que el término $d \beta_{E} / d i_{L}$ sea siempre positivo, y así procurar bajos grados de complejidad. Dadas las condiciones anteriores, se hace que $d \beta_{E} / d i_{L}=\sigma i_{L}{ }^{2}$, lo que al integrar implica que $\beta_{e}$ está dada por la ecuación 22 .

$$
\beta_{E}=\frac{1}{3} \sigma i_{L}^{3}
$$

Al haber definido la expresión $\beta_{E}$ en términos de las variables del sistema, es ahora posible construir la dinámica de $\alpha_{E}$, que previamente se condicionó para anular 
parte de la ecuación 20. Dicha condición rige su dinámica de la forma mostrada en la ecuación 23.

$$
\frac{d \alpha_{E}}{d t}=-\frac{\sigma}{L} i_{L}^{2}\left((d-1) v_{c}+\alpha_{E}+\frac{1}{3} \sigma i_{L}^{3}\right)
$$

Si se realiza un procedimiento similar, pero esta vez para obtener un estimador de la carga $(R)$, que por comodidad se trabajará en su forma de admitancia $(Y)$, se obtiene la dinámica de error de estimación de la admitancia de carga $\left(z_{Y}\right)$ dada por la ecuación 24 .

$$
\frac{d z_{Y}}{d t}=\frac{d \alpha_{Y}}{d t}+\frac{d \beta_{Y}}{d v_{c}} \frac{1}{C}\left((1-d) i_{L}-v_{c}\left(\alpha_{Y}+\beta_{Y}-z_{Y}\right)+i_{P}\right)
$$

Nuevamente, al escoger la derivada $\alpha_{Y}$ para anular todos los términos diferentes a $z_{Y}, \mathrm{y}$ asegurar estabilidad del error, se genera la ecuación 25 que muestra la dependencia de $\beta_{Y}$ a la tensión de salida.

$$
\beta_{Y}=-\rho v_{c}^{2}
$$

Mientras que $\alpha_{Y}$ presenta una evolución temporal dictada por la ecuación 26.

$$
\frac{d \alpha_{Y}}{d t}=\frac{\rho}{C} v_{c}(1-d) i_{L}+v_{c}\left(\alpha_{Y}-\rho v_{c}^{2}\right)+i_{P}
$$

Tanto para el mecanismo adaptativo de estimación de la tensión de la batería y del valor de la carga, existen dos parámetros libres $(\sigma \mathrm{y} \rho)$, cuyo valor se elige para ajustar los tiempos de asentamiento de la dinámica de los errores de estimación, que deben ser tan pequeños como sea posible para asegurar una rápida repuesta del sistema ante cambios de este. Para este caso su valor corresponde a $\sigma=2 * 10^{-3}$ y $\rho=4,5 * 10^{-3}$.

\section{RESULTADOS DE SIMULACIÓN}

En esta sección se simula el SAE con el software Matlab ${ }^{\circledR}$, para verificar el modelo realizado para el convertidor, junto con el controlador diseñado para este. En dicha simulación se usan los mismos parámetros consignados en la tabla 1, además de una frecuencia de conmutación igual a $30 \mathrm{kHz}$, mientras se somete el SAE a diferentes condiciones de operación que permitan evaluar el desempeño del controlador diseñado, cuyas ganancias se fijadas como $K_{i C}=2,5$ y $K_{i L}=0,41$, de acuerdo con el análisis hecho en la sección anterior.

La primera situación de funcionamiento planteada consiste en suplir una carga nominal de $10 \Omega$ a $48 \mathrm{~V}$ sin tener conectadas las fuentes de generación, por lo que la energía se extrae en su totalidad de las baterías del SAE. La figura 5 muestra la respuesta del sistema para esta situación, con condiciones iniciales nulas para la tensión 
y corriente en el condensador e inductor respectivamente. Con esta figura resulta evidente la condición establecida para los tiempos característicos de las variables del sistema, en la que el periodo de conmutación debe ser menor que la constante de tiempo de la variable directa de control $\left(i_{L}\right)$, y que esta a su vez, debe ser por lo menos diez veces más rápida que la variable indirecta $\left(v_{c}\right)$. Como consecuencia de esta restricción, la tensión de salida posee un ligero defecto de saturación antes de los $0,17 \mathrm{~ms}$, pero que no resulta significativo en relación con el tiempo de respuesta total del sistema. Igualmente, se debe resaltar la ausencia de sobrepicos en la respuesta de la tensión y corriente del convertidor, lo que resulta conveniente para la regulación del bus DC y la preservación de los elementos del convertidor y cargas conectadas al bus.

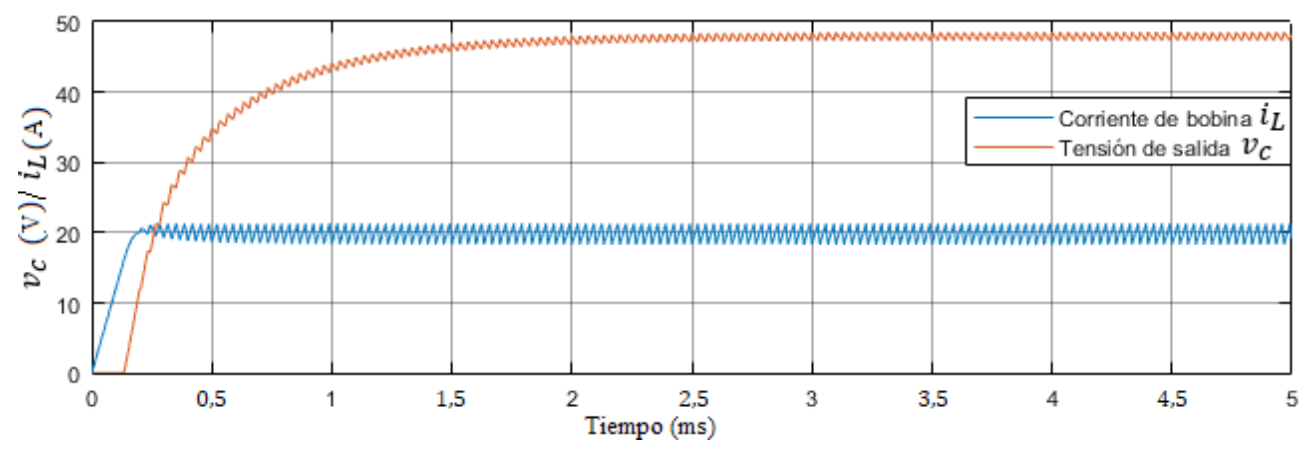

Figura 5. Respuesta transitoria de tensión de salida y corriente en el inductor del SAE

Fuente: elaboración propia

Por otra parte, para examinar el funcionamiento del mecanismo adaptativo se simula el SAE bajo las mismas condiciones que se usaron antes, pero esta vez con condiciones iniciales iguales a $48 \mathrm{~V}$ y $20 \mathrm{~A}$ para el condensador y bobina respectivamente. Además, se introducen cambios tipo paso en el valor del voltaje de la batería mientras se usa el valor nominal fijo en los bloques que contienen a las ecuaciones $3 \mathrm{y}$ 8. De la misma manera, se simula el SAE con el mecanismo adaptativo de inmersión e invariancia para estimar el valor actual de la tensión de la batería. La comparación de estos resultados se presenta en la figura 6 , en ella se ve claramente que la tensión de salida para el caso no adaptativo (arriba) tiene un error de estado estacionario, a pesar de que sigue siendo estable.

Por otro lado, al incorporar el mecanismo adaptativo, a pesar de que la tensión se desvía ligeramente del valor de referencia, después de transcurridos 2,2 $\mathrm{ms}$ el voltaje de salida retorna al valor fijado. Al comparar este valor de tiempo de establecimiento con la frecuencia de conmutación, corresponde a casi 60 periodos de conmutación, lo cual es considerable. Sin embargo, es a causa de las restricciones de diseño de las 
ecuaciones 10 y 11 , junto con las ecuaciones 13 y 14 que establecen la proporcionalidad directa entre los valores de $\mathrm{L}$ y $\mathrm{C}$, y los tiempos característicos de las variables del sistema. Ya que los valores de $\mathrm{L}$ y $\mathrm{C}$ se eligieron a partir de los bajos niveles de rizo en corriente y tensión, intentar disminuir los valores de los tiempos de asentamiento iría en detrimento de la calidad de energía de entregada por el SAE.

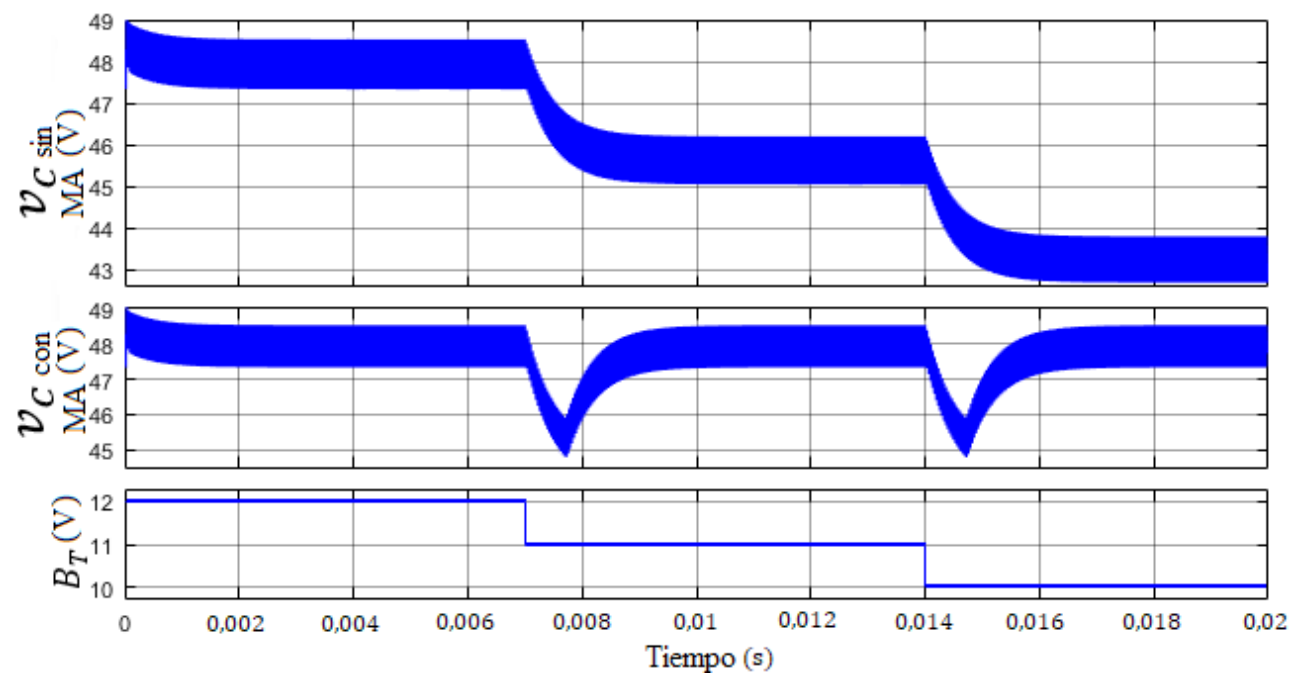

Figura 6. Tensión de salida del convertidor sin mecanismo adaptativo (arriba) y con mecanismo adaptativo (medio), frente a cambios en el valor de la tensión en la batería (abajo)

Fuente: elaboración propia

De forma similar, se somete el SAE a cambios abruptos de carga, se inicia con su valor nominal de $10 \Omega$, transformado luego a $5 \Omega$ y finalmente a $16 \Omega$. En la sección superior, la figura 7 muestra la tensión de salida ante estos cambios al no operar el mecanismo adaptativo. Entre tanto, en la sección inferior de esta se presenta la tensión de salida mientras opera el mecanismo adaptativo bajo los mismos cambios de carga. Es claro en la figura que el cambio en el valor de carga no afecta la estabilidad del sistema, pero sí el valor de referencia, lo que puede llegar a afectar el funcionamiento de la nano-red completa.

Al incorporar el mecanismo de inmersión e invariancia en el sistema se mantiene la tensión de salida en el valor de referencia, salvo un transitorio de corta duración que se presenta al inicio de la transición. La amplitud de este transitorio para dicho caso se eleva y corresponde a un $24,6 \%$ del valor final, la justificación de este valor radica en la gran magnitud de los cambios de la carga, que corresponden al 60 y $50 \%$, valores que distan considerablemente del valor nominal 


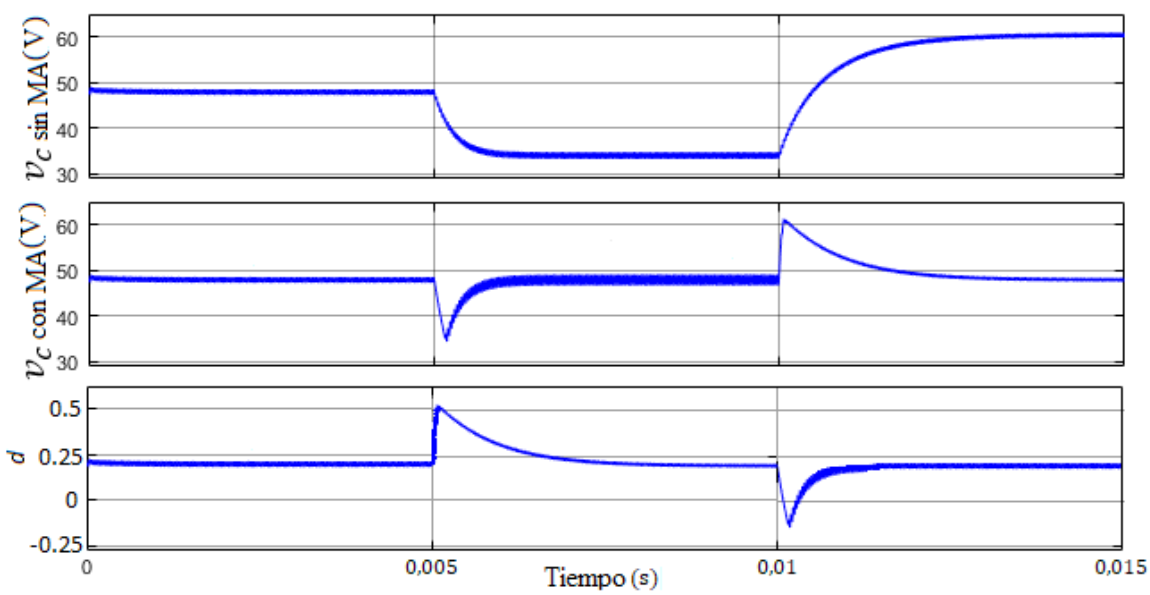

Figura 7. Tensión de salida sin mecanismo adaptativo (arriba) y con mecanismo adaptativo (abajo) frente a tres cambios de carga tipo paso

Fuente: elaboración propia

Finalmente, se simula el SAE cuando las fuentes de generación se encuentran conectadas al bus DC. Para esta simulación las condiciones iniciales de la corriente en el inductor y la tensión del condensador de salida tienen el mismo valor que para el caso anterior. Luego se somete el dispositivo diseñado a cuatro valores de corriente de las fuentes $\left(i_{P}\right)$ diferentes que tienen la misma dirección que indica la figura 3. Como resultado de lo anterior, se obtiene la figura 8 , en ella se presenta en la parte superior la tensión de salida, la corriente del inductor en la parte media y la corriente de las fuentes de generación en la parte inferior.

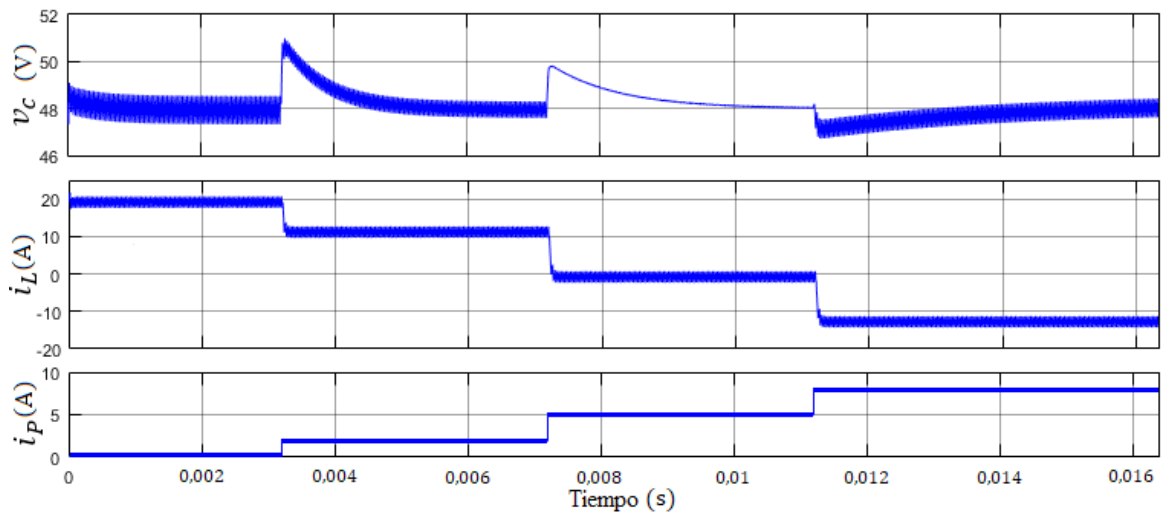

Figura 8. Tensión de salida (arriba) y corriente en el inductor (medio) frente a cambios en la corriente de las fuentes de generación (abajo) 
En la parte media de la figura 8 se observa que en la primera transición de corriente de generación, en la que pasa de 0 A a 2 A, la corriente en el inductor disminuye su valor promedio a través de una transición rápida, pero sin sobrepicos, a diferencia de la tensión de salida, la cual tiene un pico que alcanza los 50,9 V, pero que retorna a los $48 \mathrm{~V}$ transcurridos 2,2 ms. Por otra parte, cuando la corriente de generación toma el valor de $5 \mathrm{~A}$ la tensión de salida presenta nuevamente un sobrepico de características similares a la de la transición anterior, pero con un rizo mucho menor, debido a que como se puede ver en la figura, la corriente en el inductor es muy cercana a cero, por lo tanto, la energía requerida por la carga se suple en gran parte por las fuentes de la nano-red, y como estas se han supuesto ideales en su valor, el rizo asociado a ellas es nulo.

Por último, la corriente de las fuentes de generación tiene una última transición en la que toma un valor igual a $8 \mathrm{~A}$, valor que genera un excedente de energía en el bus DC, que se ha de aprovechar y almacenar en el SAE para que sea entregado en el momento en el que se requiera. Como lo muestra la figura 8 , la corriente del inductor se hace negativa para esta condición de operación, lo cual evidencia que la corriente ingresa a las baterías de SAE y estas se recargan. En esta ocasión el pico de la tensión de salida es de menor valor que para los casos anteriores y se presenta por debajo del valor de referencia. Esto debido a que el SAE se comporta ahora como una carga y extrae energía del bus DC.

\section{CONCLUSIONES}

En este documento se muestra el diseño de un sistema de almacenamiento de energía para una nano-red DC, el cual cuenta con una estrategia de control basada en pasividad, cuyas ganancias se sintonizan a partir de un criterio de márgenes de estabilidad y respuesta transitoria. Adicionalmente, se incorpora un mecanismo adaptativo que le permite al controlador estimar en tiempo real el valor de la tensión de la batería y el de la carga, solo con hacer uso del conocimiento de la dinámica del sistema y las lecturas de tensión de salida y corriente en la bobina del convertidor. Esto hace innecesaria la determinación de algunos parámetros dinámicos -como la resistencia interna de la batería- lo que permite la reducción del uso de sensores extra o técnicas complejas para obtener estos parámetros, lo que a su vez disminuye los costos de implementación.

Se presentaron los resultados de simulación del sistema desarrollado, en los que se comprueba la regulación de la tensión de salida y corriente del inductor, además de altos niveles de robustez que le permiten responder en tiempos inferiores a 2,2 ms a perturbaciones del sistema, mientras se regula la tensión de salida. Estos tiempos de respuesta dependen de las ganancias del controlador y de las constantes de ajuste del mecanismo de inmersión e invarianza. 


\section{AGRADECIMIENTOS}

El presente artículo muestra los resultados parciales del proyecto de investigación Cargador de baterías de mediana y baja capacidad con baja distorsión armónica en corriente, elevado factor de potencia y alta eficiencia para vehículos eléctricos, cuya financiación se encuentra a cargo de Colciencias (Contrato: FP44842 - 031 2016) y el Fondo Nacional para la financiación de la ciencia, la tecnología y la innovación "Fondo Francisco José de Caldas" del Departamento Administrativo de Ciencia, Tecnología e innovación, Colombia.

\section{REFERENCIAS}

[1] Sistema de información eléctrico colombiano (SIEL), Registro de proyectos de generación (Inscripción según requisitos de las Resoluciones UPME No. 0520 y No. 0638 de 2007), Unidad de Planeación Minero Energética (UPME), [En línea], Disponible: http://www.siel. gov.co/Generacion_sz/Inscripcion/2016/Registro_Proyectos_Generacion_Feb2016.pdf, 2007.

[2] M. Quintero Quintero, "Dependencia hidrológica y regulatoria en la formación de precio de la energía en un sistema hidrodominado: caso sistema eléctrico colombiano," Revistas

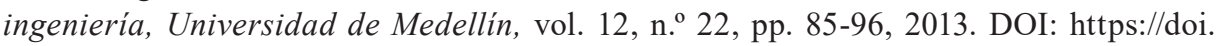
org/10.22395/rium.v12n22a7

[3] A. Cserép et al., "A nanogrid Concept for Supplying ICT Devices to Improve the Energy Efficiency of Small Offices," presentado en IEEE Second International Conference on DC Microgrids (ICDCM), Nuremberg, 2017. DOI: https://doi.org/10.1109/ICDCM.2017.8001082

[4] C. Cecati et al., "DC nanogrid for renewable sources with modular DC/DC LLC converter building block," IET Power Electronics, vol. 10, n. ${ }^{\circ}$ 5, pp. 536-544, 2017. DOI: https://doi. org/10.1049/iet-pel.2016.0200

[5] S. Ilango Ganesan et al., "Control Scheme for a Bidirectional Converter in a Self-Sustaining Low-Voltage DC Nanogrid," IEEE Transactions on Industrial Electronics, vol. 62, n. ${ }^{\circ} 10$, pp. 6327-6326, 2015. DOI: https://doi.org/10.1109/TIE.2015.2424192

[6] D. D. Campo Ossa et al., "Análisis y simulación de un rectificador trifásico controlado Active Front End (AFE)," Revistas ingeniería, Universidad de Medellín, vol. 14, n. ${ }^{\circ} 27$, pp. 257-272, 2015. DOI: https://doi.org/10.22395/rium.v14n27a15

[7] S. Ahmad Hamidi y A. Nasiri, "Stability analysis of a DC-DC converter for battery energy storage system feeding CPL," presentado en IEEE International Telecommunications Energy Conference (Intelec), Osaka, 2015. DOI: https://doi.org/10.1109/INTLEC.2015.7572343

[8] S. Oucheriah y L. Guo, "PWM-Based Adaptive Sliding-Mode Control for Boost DC-DC Converters" IEEE Transactions on Industrial Electronics, vol. 60, n. . 8, pp. 3291-3294, 2013. DOI: https://doi.org/10.1109/TIE.2012.2203769 
[9] A. Morici y Z. Tariq, "Fuzzy Logic based Adaptive Controller for AC/DC Boost Converters" presentado en International Exhibition and Conference for Power Electronics, Intelligent Motion, Renewable Energy and Energy Management, Nuremberg, Alemania, 2018.

[10] B. Wang, Y. Yuan, Y. Zhou y X. SunMotion, "Buck/Boost Bidirectional Converter TCM Control Without Zero-crossing Detection" presentado en IEEE $8^{\text {th }}$ International Power Electronics and Motion Control Conference (Ipemc-ECCE Asia), Hefei., 2016. DOI: https:// doi.org/10.1109/IPEMC.2016.7512786

[11] O. Montoya, A. Garcés, I.Ortega y G. Espinosa, "Passivity-Based Control for Battery Charging/Discharging Applications by Using a Buck-Boost DC-DC Converter" presentado en IEEE Green Technologies Conference (GreenTech), Austin, Tx., 2018. DOI: https://doi. org/10.1109/GreenTech.2018.00025

[12] M. Mojallizadeh y M. Badamchizadeh, "Adaptive Passivity-Based Control of a Photovoltaic/ Battery Hybrid Power Source via Algebraic Parameter Identification”, IEEE Journal of Photovoltaics, vol. 6, n. ${ }^{\circ}$ 2, pp. 532-539, 2016. DOI: https://doi.org/10.1109/JPHOTOV.2016.2514715

[13] D. Freitas, A. Lima y M. Morais, "Determining Lead-Acid Battery DC Resistance by Tremblay Battery Model" presentado en $7^{\text {th }}$ International Renewable Energy Congress (IREC), Hammamet, 2016. DOI: https://doi.org/10.1109/IREC.2016.7478944

[14] E. Abdulhamitbilal, "SoC Estimation for Lithium-Ion Batteries in Automotive Systems: Sliding Mode Observation" presentado en $14^{\text {th }}$ International Workshop on Variable Structure Systems (VSS), Nanjing, 2016. DOI: https://doi.org/10.1109/VSS.2016.7506936 